\title{
Segmental arterial mediolysis
}

\section{A rare cause of rapidly progressive arterial dissections}

\author{
David A. Lapides, MD; Mazen S. Abusamaan, MD; Jonathan J. Davick, MD; Aditya M. Sharma, MD; \\ James W. Mandell, MD, PhD; M. Beatriz S. Lopes, MD; J. Javier Provencio, MD; Bradford B. Worrall, MD, MSc; \\ Andrew M. Southerland, MD, MSc
}

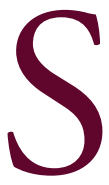

egmental arterial mediolysis (SAM) is a rare nonatherosclerotic, noninflammatory vasculopathy characterized by lytic degeneration of the arterial media, intramural dissection, and thrombosed/ruptured dissecting aneurysm. ${ }^{1}$ We present a putative case of SAM in a patient who presented with rapidly progressive systemic arterial dissections, including the cervical and cerebral vasculature, leading to devastating strokes, subarachnoid hemorrhage $(\mathrm{SAH})$, and death.

\section{Case report}

A 49-year-old woman with a history of hypertension presented to a community hospital with abdominal pain. Abdominal ultrasound suggested bilateral renal infarctions and severe renal artery stenosis. Later, she became somnolent, reported a headache and "ripping" back pain, and developed decreased movement in the legs. Pertinent neurologic examination demonstrated mild somnolence, lower extremity paraplegia with hyperreflexia, bilateral extensor responses, and a T12 spinal sensory level. ECG revealed flattened T waves and an associated troponin peak consistent with non-ST elevation myocardial infarction requiring conservative management given clinical instability. Head CT and spinal MRI revealed extensive SAH, with epidural and subdural hemorrhage from C2-T11 with medullary ischemia. CT angiography (CTA) of the head and neck revealed irregular stenoses and luminal contour in the bilateral cervical carotid and right vertebral arteries and multifocal irregularities throughout the intracranial circulation (figure). Thoraco-abdominal CTA demonstrated irregularities in the subclavian, celiac, common hepatic, bilateral renal, and external iliac arteries with intramural hematoma concerning for multifocal systemic dissections.

Laboratory serologic testing showed no supporting evidence of systemic vasculitis, hypercoagulability, or infection given negative antineutrophil cytoplasmic antibodies, extractable nuclear antigen, antinuclear antibodies, double-stranded DNA, Factor II and V Leiden mutations, protein $\mathrm{C}$ and $\mathrm{S}$ deficiency, antiphospholipid antibodies, hepatitis $\mathrm{B} / \mathrm{C}$, and cytomegalovirus antibodies. Temporal artery biopsy demonstrated no evidence of inflammation, but demonstrated prominent intimal hyperplasia compatible with fibromuscular dysplasia. She ultimately became hypotensive, requiring high-dose vasopressors, and her condition rapidly deteriorated to a comatose state with impaired brainstem reflexes. A brain MRI showed large acute infarctions in the bilateral anterior, posterior, left middle cerebral, and cerebellar arterial territories. After further goals of care discussions, she was transitioned to comfort care and died.

Postmortem examination confirmed multifocal arterial dissections of different ages including the cervical, subclavian, coronary, celiac, hepatic, renal, iliac, and small epicardial and intramedullary arteries. Arterial wall histology revealed hematoma between medial and adventitial layers with compression of the true lumen, thinning of the medial layer with degradation of

Departments of Neurology (DAL, JJP, BBW, AMS), Medicine (MSA, AMS), Pathology (JJD, JWM, MBSL), and Public Health Sciences (BBW, AMS), University of Virginia Health System, Charlottesville.

Funding information and disclosures are provided at the end of the article. Full disclosure form information provided by the authors is available with the full text of this article at Neurology.org/cp.

Correspondence to: as $5 \mathrm{ef}$ virginia.edu

\section{Practical Implications}

Consider segmental arterial

mediolysis in patients

presenting with a combination

of acute abdominal pain

and rapidly progressive

cerebrovascular vasculopathy. 


\section{Figure Radiographic-histologic correlates}
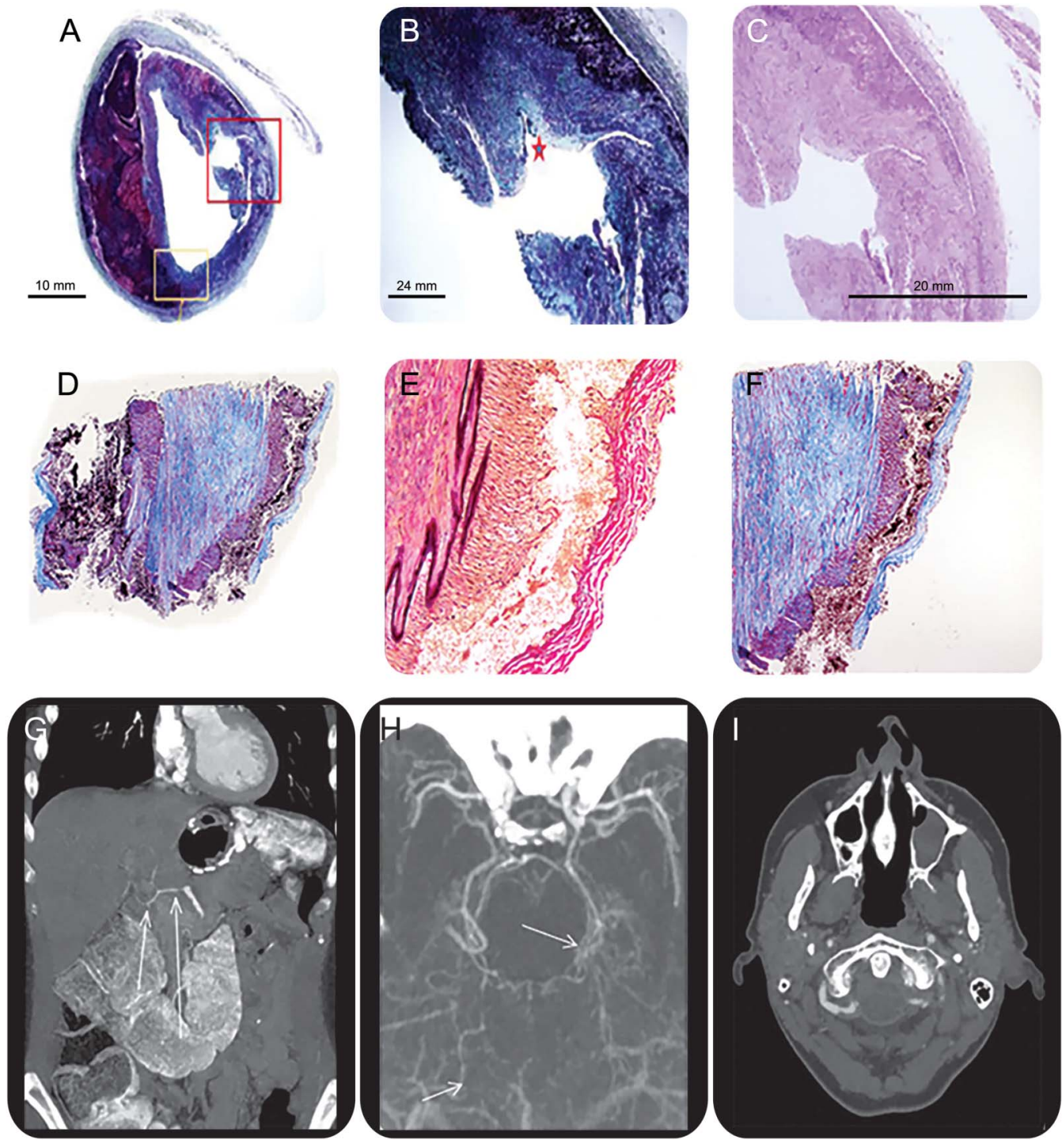

(A) Celiac arterial dissection with hematoma between media and adventitia (Movat pentachrome). (B, C) Focal loss of internal elastic laminae and thinning of media layer in celiac artery (Movat pentachrome, hematoxylin \& eosin). (D-F) Right vertebral artery with dissection between outer media and adventitial layers (Movat pentachrome, elastin, Movat pentachrome). (G) CT angiography demonstrates celiac artery dissection. (H) CT angiography head shows left middle cerebral artery stenosis and diffuse irregularity. (I) CT angiography shows right vertebral irregular tapering.

smooth muscle fibers, collagenous loss, and focal elastic breaks with breakdown of the elastic laminae. There was no evidence of vasculitis or atherosclerosis. Presence of foamy or vacuolar degeneration of the smooth muscle in the outer media was considered to be most supportive of segmental arterial mediolysis.

\section{DISCUSSION}

SAM typically presents late in the fifth decade, manifesting with abdominal pain due to visceral artery dissections and hemorrhage. ${ }^{2}$ The clinical syndrome is rapidly progressive with a high mortality rate $(25 \%-50 \%)$ due to multifocal degeneration of the arterial wall, leading to dissections and aneurysm formation. ${ }^{3}$ Approximately $18 \%$ of cases involve the cerebrovasculature. ${ }^{3}$ With increases in the use of noninvasive vascular imaging, the diagnosis of SAM has been more widely reported in the last decade. 
After proceeding to autopsy, the pathologic differential included SAM, fibromuscular dysplasia (FMD), and cystic medial necrosis (CMN). ${ }^{4}$ SAM is histologically similar to FMD with loss of smooth muscle integrity and intimal proliferation. However, these entities are clinically distinct as FMD often has a favorable prognosis compared to the rapid clinical deterioration in SAM. In addition, FMD shows alternating areas of thinned media and thickened fibromuscular ridges containing increased collagen, not seen in our case. Finally, CMN was unlikely given absence of basophilic ground substance and cystic lesions in the media.

Shared genetic associations in FMD and SAM remain poorly defined and further research is needed to investigate environmental triggers of connective tissue vasculopathies. Of interest, independent genomic analyses have recently discovered a common risk allele for both FMD and cervical artery dissection in the PHACTR1 gene on chromosome 6.5, $\mathrm{In}$ our case, multifocal arterial dissections in the cervical and intracranial vasculature contributed to neurologic decline, with intracranial extension of the right vertebral artery dissection causing SAH. Of note, the intracranial circulation lacks an external elastic lamina, making it prone to arterial rupture in the setting of cerebral vasculopathy. ${ }^{7}$ A remarkable aspect of this patient's disease was the rapid pace with which it progressed from initial symptoms to death over several days. This rapidly progressive phenotype may represent a severe variant along the spectrum of SAM. Neurologists should be aware of this uncommon devastating syndrome in a patient presenting with both cerebrovascular and visceral vasculopathy. Earlier recognition of SAM may lead to prompt management and better outcomes.

\section{REFERENCES}

1. Lie J. Segmental mediolytic arteritis: not an arteritis but a variant of arterial fibromuscular dysplasia. Arch Pathol Lab Med 1992;116:238-241.

2. Chao CP. Segmental arterial mediolysis. Semin Intervent Radiol 2009;26:224-232.

3. Shenouda M, Riga C, Naji Y, et al. Segmental arterial mediolysis: a systematic review of 85 cases. Ann Vasc Surg 2014;28:269-277.

4. Olin JW, Gornik HL, Bacharach JM, et al. Fibromuscular dysplasia: state of the science and critical unanswered questions: a scientific statement from the American Heart Association. Circulation 2014; 129:1048-1078.

5. Kiando SR, Tucker NR, Castro-Vega LJ, et al. PHACTR1 is a genetic susceptibility locus for fibromuscular dysplasia supporting its complex genetic pattern of inheritance. PLoS Genet 2016;12: e1006367.

6. Debette S, Kamatani Y, Metso TM, et al. Common variation in PHACTR1 is associated with susceptibility to cervical artery dissection. Nat Genet 2015;47:78-83.

7. Southerland A, Meschia J, Worrall B. Shared associated of nonatherosclerotic, large vessel, cerebrovascular arteriopathies: considering intracranial aneurysms, cervical artery dissection, moyamoya disease and fibromuscular dysplasia. Curr Opin Neurol 2013;26:13-28.

\section{AUTHOR CONTRIBUTIONS}

D.A. Lapides: drafting of manuscript. M.S. Abusamaan: drafting of manuscript. J.J. Davick: pathologic analysis and interpretation. A.M. Sharma: critical revision of manuscript for clinical and intellectual content. J.W. Mandell: pathologic analysis and interpretation. M.B.S. Lopes: pathologic analysis and interpretation. J.J. Provencio: critical revision of manuscript for clinical and intellectual content. B.B. Worrall: critical revision of manuscript for clinical and intellectual content. A.M. Southerland: drafting of manuscript, critical revision of manuscript for clinical and intellectual content.

\section{STUDY FUNDING}

No targeted funding reported.

\section{DISCLOSURES}

D.A. Lapides, M.S. Abusamaan, and J.J. Davick report no disclosures. A.M. Sharma serves as CME Editor for Vascular Medicine. J.W. Mandell serves as Associate Editor for Journal of Neuropathology and 
Experimental Neurology; is author on a patent re: SIMPLE: Sequential Immunoperoxidase Labeling and Erasing Technology for multiplex immunohistochemistry; receives research support from NIH; and receives license fee payments for FIRM - fluorescent stain for resin sections from Electron Microscopy Sciences, Inc. M.B.S. Lopes serves on the editorial boards of Journal of Neuropathology and Experimental Neurology, American Journal of Surgical Pathology, and Acta Neuropathologica Communications. J.J. Provencio serves on a scientific advisory board for and received funding for travel from Minnetronix, Inc.; has received funding for travel and speaker honoraria from Bard, Inc.; serves on the editorial board of Journal of Neuroimmunology; receives publishing royalties for An Intensive Care Guide for the Family: A Resource for Families of Patients in the Intensive Care Unit (Createspace, 2014); and receives research support from Bard Inc. and NIH/NINDS. B.B. Worrall serves as Deputy Editor of Neurology ${ }^{\circledR}$; receives publishing royalties for Merritt's Neurology editions 10, 11, and 12 (Lippincott Williams \& Wilkins, 2009); and receives research support from NIH (NINDS, NHGRI) and University of Virginia-Centers of Excellence. A.M. Southerland serves as Deputy Podcast Editor for Neurology; is author on a patent re: Method, System and Computer Readable Medium for Improving Treatment Times for Rapid Evaluation of Acute Stroke via Mobile Telemedicine; has provided expert review in medico-legal cases; and receives research support from Claret Medical, Edwards Lifesciences, NIH (NINDS, NHLBI), American Heart Association-American Stroke Association, American Academy of Neurology, and American Board of Psychiatry and Neurology. Full disclosure form information provided by the authors is available with the full text of this article at Neurology.org/cp.

\section{Enjoy Neurology ${ }^{\circledR}$ Clinical Practice on your iPad ${ }^{\circledR}$}

The same information so critical to your practice is now brought to you on the $\mathrm{iPad}^{\circledR}$. This dynamic app optimizes the best in digital technology to enhance the reading experience with article-sharing features, multimedia, links, and more.

\section{Enjoy the benefits:}

- Easy-to-read articles you can share via email and social media

- Adjustable text sizing with "pinch and zoom" technology

- Engaging multimedia videos, images, and data supplements

- Ability to store downloaded issues

- Convenient notification when a new issue is available

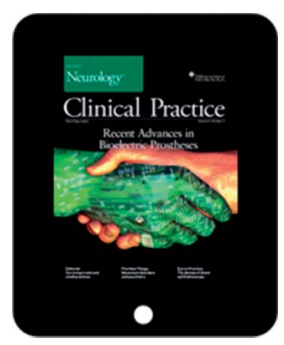

AAN members - enjoy Full Access to every issue on your iPad ${ }^{\circledR}$. If you are not yet a member, download the app and GET THE LATEST ISSUE FREE! (Go to http://bit.ly/NCPapp for details.) 